ANL-HEP-CP-97-66

For the Proceedings of the Conference on

Beyond the Standard Model V

29 April - 02 May 1997, Balholm, Norway

$$
\text { CONF-9704155-- }
$$

\title{
Recent Electroweak Measurements from the Tevatron
}

\author{
Robert G. Wagner \\ For the CDF \& DØ Collaborations
}

High Energy Physics Division

Argonne National Laboratory ${ }^{1}$

Argonne, Mlinois 60439-4815

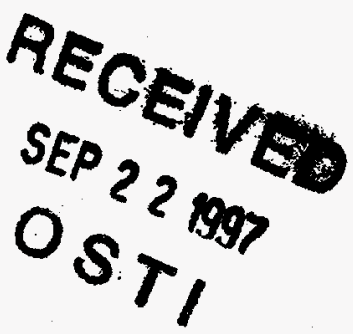

\begin{abstract}
Preliminary electroweak results are discussed from the Fermilab experiments, CDF and DØ, based on Tevatron run $\mathrm{lb}$ data. These include an updated precision measurement of the $W$ mass which when combined with previous Tevatron, CERN Sp̄pS, and LEP-II results gives a combined world average $M_{W}=80.40 \pm 0.08 \mathrm{GeV} / \mathrm{c}^{2}$. Also presented are new limits on anomalous gauge boson self-couplings, measurement of the $W$ charge asymmetry, $\sigma \cdot B\left(W \rightarrow \tau \nu_{r}\right)$, and limits on quark/lepton compositeness from high mass Drell-Yan production.
\end{abstract}

\section{INTRODUCTION}

The CDF and $D \emptyset$ collaborations have recently produced measurements of the $W$ mass, $M_{W}$, based on integrated lumirosities of $80 \mathrm{pb}^{-1}$ (D $\emptyset$ ) and $90 \mathrm{pb}^{-1}$ (CDF) of data collected from run lb of the Fermilab Tevatron collider. While precision measurement of $M_{W}$ tests the Standard Model beyond the lowest order through radiative corrections to the mass, of more current interest is the simultaneous measurement of $M_{W}$ and $M_{\text {top }}$ and the constraint placed on the Higgs boson mass via loop corrections $\left(\Delta M_{W} \propto M_{\text {top }}^{2}, \ln M_{\text {Higgs }}\right)$. In the next section I review the CDF and $D \emptyset W^{r}$ mass analysis and present both the preiiminary run $1 \mathrm{~b}$ results and the combined $M_{W}$ estimate based on CDF, $D \varnothing$, UA2, and LEP-II.

Additional electroweak physics results have been made availabie recently in preliminary form by $\mathrm{D} \emptyset$ and $\mathrm{CDF}$. In sections subsequent to the $W$ mass review, I present results from $D \emptyset$ on gauge boson self-couplings from analysis of gauge boson pair production and on the $W \rightarrow \tau \nu_{r}$ branching ratio; and from CDF on the $W\left(\rightarrow e \nu_{e}, \mu \nu_{\mu}\right)$ charge asymmetry and on high mass

Work supported in part by the U.S. Department of Energy,

Division of High Energy Physics, Contract W-31-109-ENG-38. 


\section{DISCLAIMER}

This report was prepared as an account of work sponsored by an agency of the United States Government. Neither the United States Government nor any agency thereof, nor any of their employees, makes any warranty, express or implied, or assumes any legal liability or responsibility for the accuracy, completeness, or usefulness of any information, apparatus, product, or process disclosed, or represents that its use would not infringe privately owned rights. Reference herein to any specific commercial product, process, or service by trade name, trademark, manufacturer, or otherwise does not necessarily constitute or imply its endorsement, recommendation, or favoring by the United States Government or any agency thereof. The views and opinions of authors expressed herein do not necessarily state or reflect those of the United States Government or any agency thereof. 


\section{DISCLAMMER}

Portions of this doement myy be illegible in electronic image products. Images are produced from the best available original docomenert 
Drell-Yan lepton pair production with implications for limits on quark/lepton compositeness [1].

\section{CDF AND D $\emptyset M_{W}$ MEASUREMENTS}

Precision measurement of the $W$ mass is accomplished by analysis of $W \rightarrow e \nu_{e}$ or $\mu \nu_{\mu}$. In $\bar{p} p$ collisions, many of the particles are produced in the uninstrumented angular regions near the beam pipe which prevents measurement of the longitudinal momentum balance. With the preclusion of a direct reconstruction of the $W$ mass, $C D F$ and $D \emptyset$ rely on a model, first developed by the UA2 collaboration [2], that begins with measuring the components of momenta transverse to the beam axis and inferring the unobserved transverse energy of the decay neutrino, $E_{\mathrm{T}}^{\nu}$, from the $E_{\mathrm{T}}$ balance of the charged lepton, $\overrightarrow{\mathrm{E}}_{\mathrm{T}}^{\ell}$, and the system recoiling from the $W, \overrightarrow{\mathrm{u}}$ :

$$
\overrightarrow{\mathbf{E}}_{\mathrm{T}}^{\nu}=-\left(\overrightarrow{\mathbf{E}}_{\mathrm{T}}^{\ell}+\overrightarrow{\mathrm{u}}\right)
$$

The "transverse mass" of the $W$ is then calculated from

$$
\left(M_{\mathrm{T}}^{W}\right)^{2}=\left(E_{\mathrm{T}}^{\ell}+E_{\mathrm{T}}^{\nu}\right)^{2}-\left(\overrightarrow{\mathrm{E}}_{\mathrm{T}}^{\ell}+\overrightarrow{\mathrm{E}}_{\mathrm{T}}^{\nu}\right)^{2}=2\left|p_{\mathrm{T}}^{\ell} \| p_{\mathrm{T}}^{\nu}\right|\left(1-\cos \phi_{\ell \nu}\right) .
$$

Finally, $M_{W}$ is extracted from a likelihood fit performed on the $M_{\mathrm{T}}^{W}$ lineshape by comparing data to Monte Carlo distributions generated for a range of $M_{W}$.

While CDF uses both the muon and electron decay modes for the precision measurement, the Tevatron run $1 \mathrm{~b}$ electron channel mass analysis is still in progress and only results from the muon channel are presented here. $D \emptyset$ uses only the electron channel for the $M_{W}$ measurement. Descriptions of the detectors are found in references $[3,4]$. For CDF, the muon momentum and direction are determined from reconstruction of the track in the Central Tracking Chamber (CTC) which is contained in a $1.4 \mathrm{~T}$ solenoidal magnetic field. The $D \varnothing$ experiment determines the electron direction using the EM calorimeter cluster centroid and the associated track reconstructed in the tracking chamber and the energy is measured by calorimetry with the scale set by mass reconstruction of $\pi^{\circ} \rightarrow \gamma \gamma, \psi \rightarrow e^{+} e^{-}$, and $Z \rightarrow e^{+} e^{-}$.

CDF selects $\sim 21000 W \rightarrow \mu \nu_{\mu}$ events having $25 \leq\left(p_{\mathrm{T}}^{\mu} \& \mathrm{E}_{\mathrm{T}}\right) \leq 60$ $\mathrm{GeV} / \mathrm{c}$ and recoil $|\overrightarrow{\mathbf{u}}| \leq 20 \mathrm{GeV}$. The muons must be minimum ionizing in the calorimetry and match with a stub segment in the muon drift chambers located radially outside the central calorimetry. Additional requirements are made to remove cosmic rays and $Z$ events that were mis-identified due to having a muon in a region devoid of tracking. Finally, $M_{\mathrm{T}}^{W}$ is required to be in the range $50-120 \mathrm{GeV} / \mathrm{c}^{2}$. The $M_{\mathrm{T}}^{W}$ fit region of $65-100 \mathrm{GeV} / \mathrm{c}^{2}$ contains $\sim 15000$ events.

DØ obtains 28323 events for $W \rightarrow e \nu_{e}$ requiring $\left(p_{\mathrm{T}}^{e} \& \AA_{\mathrm{T}}\right)>25 \mathrm{GeV}$ and $|\overrightarrow{\mathbf{u}}|<15 \mathrm{GeV} / \mathrm{c}$. Electrons are required to have an EM calorimeter cluster 


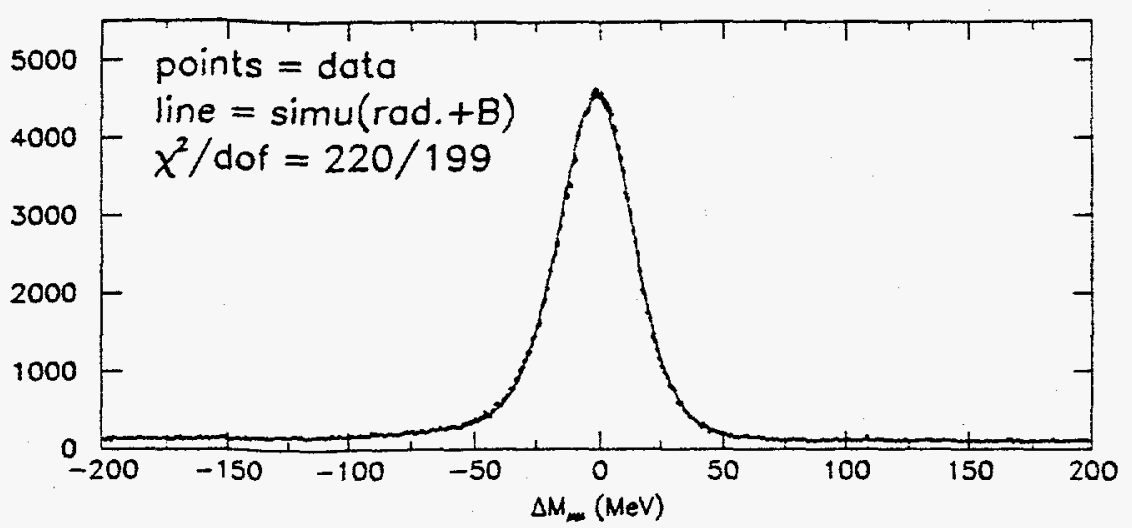

FIGURE 1. The mass difference between $J / \psi \rightarrow \mu^{+} \mu^{-}$observed in the CDF tracking chamber and the world average $M_{\psi}$. Data are represented by points and the solid curve is the best fit to a simulated lineshape including contributions from radiative decay of the $J / \psi$ and non-prompt $J / \psi$ production in $B$-meson decays.

that matches with a track in the central detector, an electron-like shower shape, and the energy in an annular ring of $\Delta R \equiv \sqrt{(\Delta \eta)^{2}+(\Delta \phi)^{2}}=0.2-0.4$ centered on the electron must be less than $15 \%$ of the electron energy.

Both experiments also select $Z$ event samples for energy/momentum scale calibration and checking.

The CDF $M_{W}$ measurement begins with a determination of the CTC momentum scale by fitting the $J / \psi \rightarrow \mu^{+} \mu^{-}$mass spectrum obtained from a sample of $\sim 250,000$ events and comparing to the world average value. The reconstructed muon momentum is corrected for energy lost during traversal of the detector; run-to-run magnetic field variation; and geometric misalignment of the magnet, beam, and CTC. Figure 1 shows the difference between the reconstructed $J / \psi$ mass and the world average value. The measured $M_{\psi}$ is $3096.2 \pm 1.5 \mathrm{MeV} / \mathrm{c}^{2}$ with the dominant contributions to the uncertainty attributed to knowledge of muon energy loss in the detector $(1.0 \mathrm{MeV})$ and possible variation of the momentum scale with increasing muon transverse momentum $(1.0 \mathrm{MeV})$. Comparison to the Particle Data tables $M_{\psi}[5]$ gives a momentum scale correction factor, $1.00023 \pm 0.00048$. The uncertainty of $\pm 1.5 \mathrm{MeV} / \mathrm{c}^{2}$ extrapolated from $M_{\psi}$ to $M_{W}$ implies a contribution from momentum scale of $\delta M_{W}= \pm 40 \mathrm{MeV} / \mathrm{c}^{2}$.

$D \emptyset$ parametrizes the energy measured in the EM calorimeter as

$$
\begin{gathered}
E_{\text {meas }}=\alpha_{\mathrm{EM}} E_{\text {true }}+\delta_{\mathrm{EM}} \\
\alpha_{\mathrm{EM}} \equiv \text { energy scale factor, } \delta_{\mathrm{EM}} \equiv \text { offset }
\end{gathered}
$$

Measured values of $\pi^{\circ} \rightarrow \gamma \gamma$ and $J / \psi, Z^{\circ} \rightarrow e^{+} e^{-}$masses are linear in the coefficients, $\alpha_{\mathrm{EM}}$ and $\delta_{\mathrm{EM}}$, and provide intersecting elliptical constraints on 

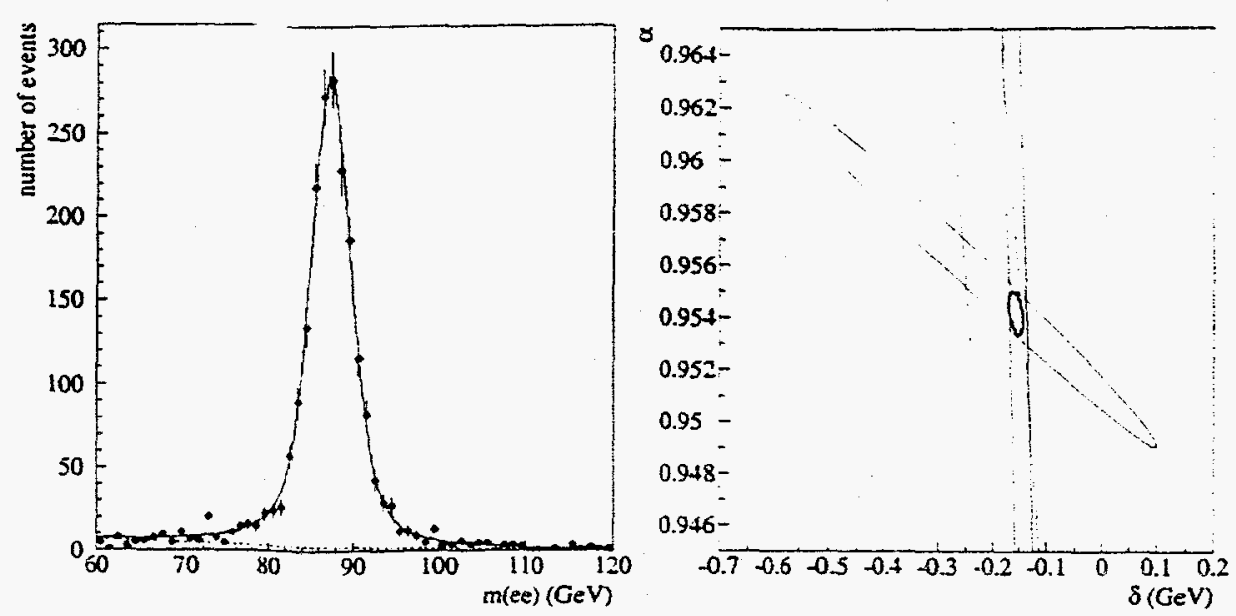

FIGURE 2. Fitted Dø $Z$ mass used for energy scale calibration (left). The $\pi^{\circ}$ and $J / \psi$ mass plots are not shown. Elliptical constraints on EM scale calibration parameters, $\alpha_{\mathrm{EM}}$ and $\delta_{\mathrm{EM}}$ based on measurements of $\pi^{\circ}, J / \psi$, and $Z^{\circ}$ masses (right).

them (see figure 2). The values for the parameters extracted using the data of figure 2 are $\alpha_{\mathrm{EM}}=0.95372 \pm 0.00091$ and $\delta_{\mathrm{EM}}=-0.16_{-0.21}^{+0.03} \mathrm{GeV}$.

The components of the recoil model are the $W$ transerve momentum, $p_{\mathrm{T}}^{W}$, and the hadronic recoil response of the calorimetry. Both experiments use the next-to-leading order calculation of $p_{T}^{W}$ due to Arnold and Kauffman [6] supplemented by a low $p_{T}^{W}$ parametrization of Ladinsky and Yuan [7].

$\mathrm{CDF}$ derives the response function using the recoil hadron-dielectron momentum balance for a sample of $Z$ events and the resolution function is determined from minimum bias trigger data. The adequacy of the model is checked using the recoil otserved in $Z$ data and the $u_{\perp}$ distribution from the $W$ data. The $u_{\perp}$ axis is defined as that perpendicular to the $W$ decay muon.

Similariy, DØ extracts both the response and resolution functions from $Z \rightarrow$ $e^{+} e^{-}$data. Recoil components along the angular bisector of the $e^{+} e^{-}$pair are added as are those normal to the bisector. The response and resolution are tuned via Monte Carlo simulation to reproduce the observed distributions.

$W$ events for a range of $M_{W}$ are generated and processed through detector simulations. Likelihood values are calculated comparing to the observed $M_{\mathrm{T}}^{W}$ distribution and $M_{W}$ is extracted from a fit to the loglikelihood function. The $M_{\mathrm{T}}^{W}$ distributions with the best fits overlaid are shown in figure 3 . Table 1 gives the systematic uncertainty contributions for both measurements. The preliminary Tevatron run $1 \mathrm{~b}$ values are $M_{W}(\mathrm{CDF})=80.430 \pm 0.100$ (stat) \pm 0.155 (syst) $\mathrm{GeV} / \mathrm{c}^{2}$ and $M_{W}(\mathrm{D} \varnothing)=80.45 \pm 0.10$ (stat) \pm 0.07 (syst) $\mathrm{GeV} / \mathrm{c}^{2}$. The $\mathrm{D} \emptyset$ statistical uncertainty is a combination of $\pm 70 \mathrm{MeV} / \mathrm{c}^{2}$ from the fit and $\pm 65 \mathrm{MeV} / \mathrm{c}^{2}$ from normalization to the world average $Z$ mass. CDF expects to improve the systematic uncertainty somewhat as the recoil model analysis is improved. A 

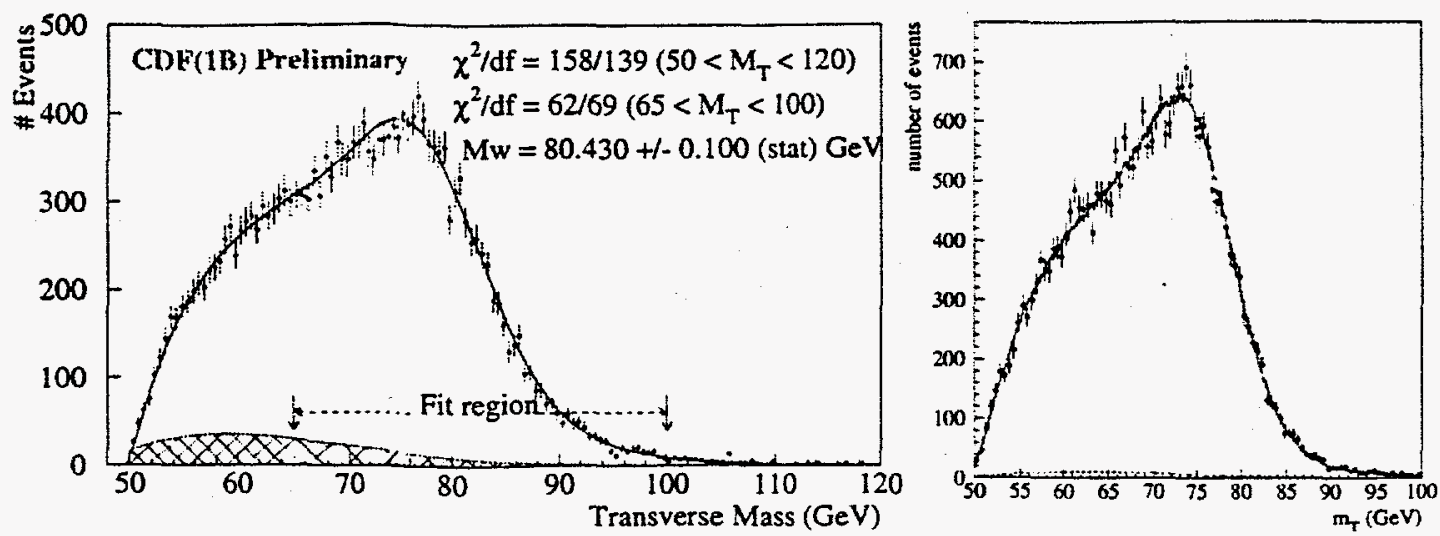

FIGURE 3. The CDF(left) and $\mathrm{D} \emptyset$ (right) $W$ transverse mass distributions (points) with best fits from Monte Carlo overlaid (solid line). Cross-hatched (CDF) and dotted (DØ) curves indicate the combined background from $W \rightarrow \tau \nu_{\tau}$, mis-identified $Z_{\mathrm{s}}$, QCD di-jets, and, for the CDF muon channel, residual cosmic ray events.

summary of precision $W$ mass measurements is given in figure 4 along with the corresponding constraint on $M_{\text {Higgs }}$ provided by $M_{W}$ vs $M_{\text {top. }}$.

\section{GAUGE BOSON SELF-COUPLINGS}

Electroweak theory specifies all fermion and gauge boson couplings. While the coupling of leptons and quarks to gauge bosons has been well-measured, the gauge bosons' couplings to themselves is not yet verified to be that of the Standard Model. At the Tevatron all combinations of pair production of $W^{ \pm}, Z^{\circ}$, and $\gamma$ are kinematically accessible allowing measurement of standard tri-linear couplings such as $W W Z$ and $W W \gamma$ as well as searches for nonstandard couplings such as $Z Z \%$.

$D \varnothing$ has completed analysis of $W \gamma$ production for all of Tevatron run 1a + run 1b. Event selection requires a high $E_{\mathrm{T}}$ charged lepton ( $\mu$ or $e$ ), $\mathrm{E}_{\mathrm{T}}>25 \mathrm{GeV}$, and a photon with $E_{\mathrm{T}} \geq 10 \mathrm{GeV}$. The radiative $W$ decay contribution is reduced relative to direct production from the $W$ propagator by requiring the lepton-photon angular separation to be greater than 0.7 . The main background is from $W+j e t s$ in which the fragmentation of one of the jets fakes a photon signature. The background-subtracted number of $W \gamma$ events is $84_{-11}^{+12}$ (stat) \pm 9 (syst), giving a cross section

$$
\sigma(\bar{p} p \rightarrow W \gamma \rightarrow \ell \nu \gamma)=11.3_{-1.5}^{+1.7}(\text { stat }) \pm 1.5(\text { sys }) \mathrm{pb}
$$

Std. Model $: \sigma(\bar{p} p \rightarrow W \gamma \rightarrow \ell \nu \gamma)=12.5 \pm 1.0 \mathrm{pb}$

Figure 5 shows the photon $E_{\mathrm{T}}, \ell-\gamma$ angular separation, and $W \gamma$ minimum invariant (also called transverse cluster) mass. A binned likelihood fit to the 
TABLE 1. Statistical and systematic uncertainty contributions for the preliminary run $1 \mathrm{~b} C D F$ and $\mathrm{D} \emptyset \mathrm{W}$ mass measurements

\begin{tabular}{llcc} 
& Preliminary Uncertainty & $\begin{array}{c}\Delta M_{W}^{\mu} \mathrm{MeV} / \mathrm{c}^{2} \\
\mathrm{CDF} \mathrm{Ib}\end{array}$ & $\begin{array}{c}\Delta M_{W}^{e} \mathrm{MeV} / \mathrm{c}^{2} \\
\mathrm{D} \emptyset \mathrm{lb}\end{array}$ \\
\hline I. $\quad$ Statistical & 100 & $70 \oplus 65$ \\
\hline II. & Momentum Scale & 40 & - \\
& Calorimeter linearity & - & 20 \\
& Electron polar angle & - & 28 \\
\hline III. $\quad$ Other Systematics & 115 & 60 \\
\hline & 1. Resolution & 25 & 23 \\
2. Input $p_{\mathrm{T}}^{W}$ & 40 & 5 \\
3. Recoil modeling & 90 & 39 \\
4. Parton dist. fcn. & 25 & 21 \\
5. Selection bias & 10 & - \\
6. Trigger bias & 15 & - \\
7. Radiative corr. & 20 & 20 \\
8. Higher-order corr. & 20 & - \\
9. Backgrounds & 25 & 12 \\
10. Fitting & 10 & - \\
11. W width & - & 9 \\
12. Calorimeter uniformity & - & 10 \\
13. Parton luminosity & - & 10 \\
14. Lepton removal & - & 16 \\
Total systematics & 122 & 118 \\
\hline TOTAL UNCERTANTY & 155 & \\
\hline
\end{tabular}
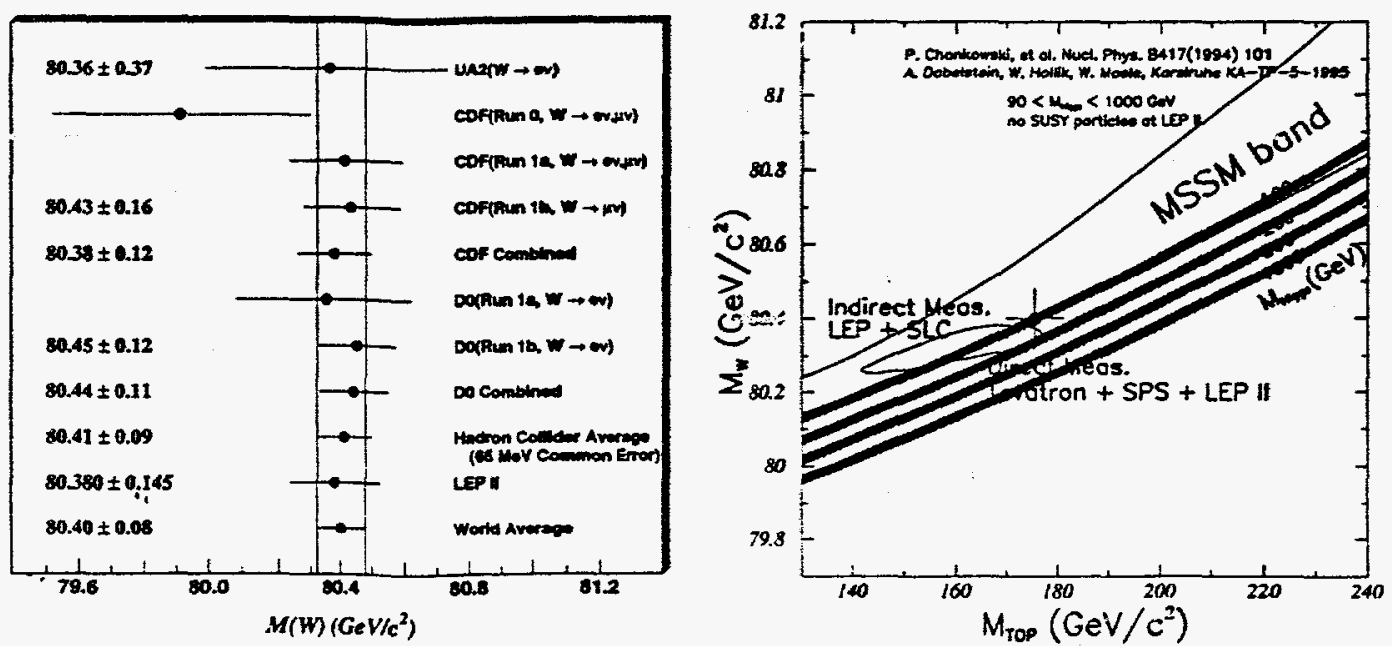

FIGURE 4. Summary of existing precision $M_{W}$ measurements (left). The righthand plot shows the combined measurement for $M_{W}$ vs. $M_{\text {top }}$ with $M_{\text {Higgs }}$ bands from loop corrections overlaid. 

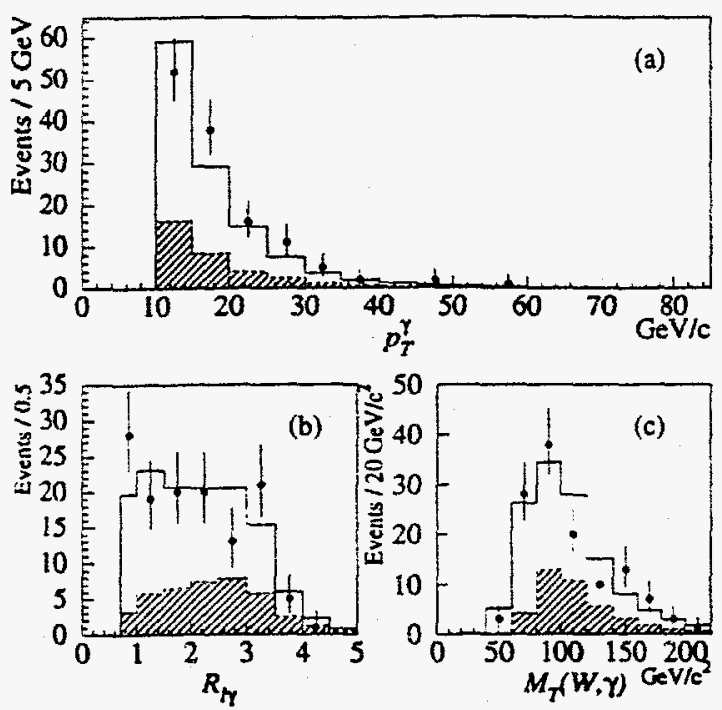

FIGURE 5. From the $\mathrm{D} \emptyset W \gamma$ analysis are shown the (a) photon $p_{\mathrm{T}}$ spectrum, (b) charged lepton-photon angular separation, and (c) $W \gamma$ minimum invariant mass. Shaded regions indicate estimated background and the open histogram is the background plus Standard Model expectation.

photon $E_{\mathrm{T}}$ spectrum is used to provide 2-d contour limits on anomalous $W W \gamma$ couplings, $\Delta \kappa_{\gamma}$ and $\lambda[8]$. One dimensional limits are derived by setting one of the couplings to zero and extracting the $95 \%$ c.l. for the other from the likelihood function. Thus,

$$
\left.\begin{array}{lr}
-0.93<\Delta \kappa_{\gamma}<0.94 & (\text { for } \lambda=0) \\
-0.31<\lambda<0.29 & \text { (for } \left.\Delta \kappa_{\gamma}=0\right)
\end{array}\right\} 95 \% \text { c.l. }
$$

$\mathrm{D} \varnothing$ and CDF have searched for $Z \gamma$ cuupling, a phenomenon not present in Electroweak theory. Run 1a results have been published and CDF has previously presented preliminary results from run $1 \mathrm{~b}$ based on $67 \mathrm{pb}^{-1}$ partial sample. From all run $1 b$ data, $D \emptyset$ has produced in preijminary form a search for $\bar{p} p \rightarrow Z\left(\rightarrow e^{+} e^{-}\right)+\gamma+X$ selecting events in a similar manner to those of $W \gamma$ except there is no $\mathcal{E}_{\mathrm{T}}$ requirement and $M\left(e^{+} e^{-}\right)$must be consistent with $M_{Z}$. For the CP-conserving couplings, $h_{30}^{Z}$ and $h_{40}^{Z}$ [9], 2-d limits are shown in figure 6 . The unitarity limit is particularly sensitive to the form factor scale used and has been set to $\Lambda_{\mathrm{FF}}=500 \mathrm{GeV}$ for all limits shown in the figure with the exception of that for L3. The most stringent limit derives from the $D \varnothing$ run 1a analysis of the mode $Z(\rightarrow \nu \bar{\nu})+\gamma$ which has the advantage of having no competing radiative decay mode and is sensitive to all neutral decays of the $Z^{\circ}$. The photon $E_{\mathrm{T}}$ requirement was raised to $E_{\mathrm{T}}^{\gamma}>40 \mathrm{GeV}$ to remove background from $W \rightarrow e \nu_{e}$ events where the electron is mis-identified as a photon due to lack of an associated track. Since anomalous couplings mainly 


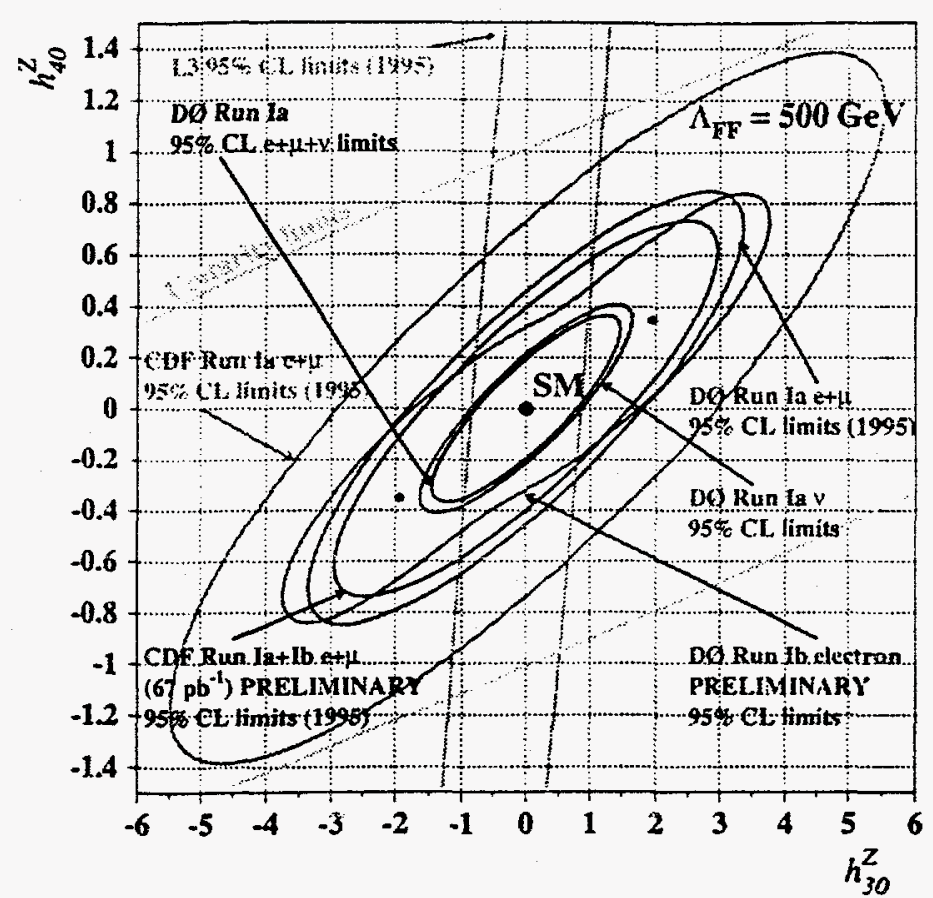

FIGURE 6. Two dimensional contour limits for anomalous CP-conserving couplings for $Z Z \gamma$. See text for explanation of form factor scale and other details.

enhance the high $E_{\mathrm{T}}^{\gamma}$ region, this has little effect on the coupling limit. From analyses of $W W$ and $W Z$ production, limits on $\Delta \kappa_{Z, \gamma}$ and $\lambda_{Z, \gamma}$ have been obtained for both $D \varnothing$ (preliminary run 1b) and CDF (final run 1b). These are not covered here due to space limitations. The public Electroweak Web pages [1] for both groups contain full details of the analyses which provide limits comparable to those presented above for $W \gamma$.

\section{CDF $W$ CHARGE ASYMMETRY}

Due to parity violation in the weak interaction a forward/backwaid asymmetry occurs in $W \rightarrow \ell \nu_{\ell}$. CDF has measured the asymmetry between $W^{+}$ and $W^{-}$as a function of the charged lepton pseudorapidity using both the electron, and muon decay channels. Because the proton $u$-quark parton distribution function, $u(x)$, is greater than that of the $d$-quark, $d(x)$, the $W^{+}\left(W^{-}\right)$ is boosted along the $p(\bar{p})$ direction and the observed asymmetry is most sensitive to the choice of PDF. This PDF constraint has proved useful in reducing the systematic uncertainty contribution to the $W$ mass.

Although 3-d track reconstruction using the CTC is unavailable beyond $|\eta| \sim 1.2$, the measurement has been extended to $|\eta| \simeq 2.0$ by using the expected correspondence between a track in the CDF silicon microtracker and 


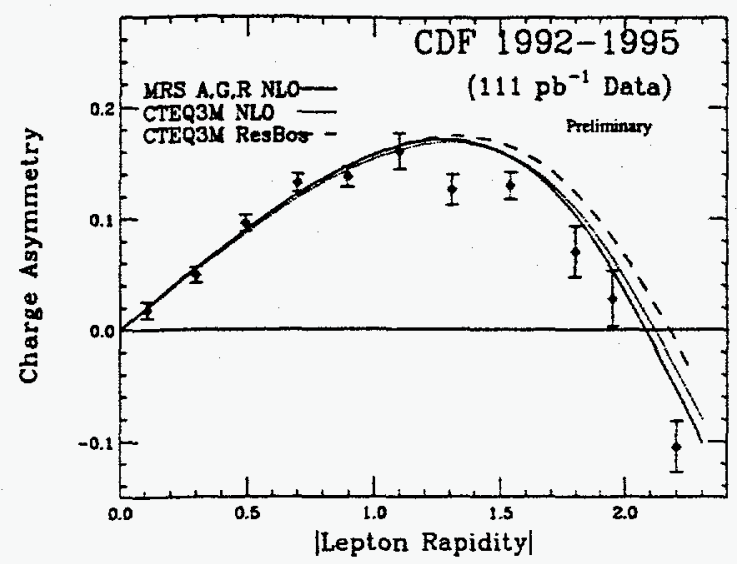

FIGURE 7. The charged lepton asymmetry, as measured by CDF, between $\mathrm{W}^{+}$and $\mathrm{W}^{-}$ as a function of charged lepton pseudorapidity.

the plug EM calorimeter. A point at $|\eta|=2.2$ is provided via the CDF forward muon detector. Results are shown in figure 7 along with expectations due to various choices of PDF.

$$
\text { V } \mathbf{D} \varnothing \sigma \cdot B\left(W \rightarrow \tau \nu_{\tau}\right)
$$

$D \varnothing$ has improved the previous CDF measurement [10] of the cross section times branching ratio for $W \rightarrow \tau \nu_{\tau}$ using the hadronic decay of the $\tau$ which is identified as an isolated narrow jet. Events selected had $E_{\mathrm{T}}(j e t)>25 \mathrm{GeV}$ $(|\eta|<0.9)$ and $E_{T}>25 \mathrm{GeV}$. Signal and background were estimated from the distribution of a variable designated, Profile $\equiv$ (sum of highest two tower $\left.E_{\mathrm{T}}\right) /\left(\right.$ cluster $\left.E_{\mathrm{T}}\right)$. Profile $<0.35$ was designated the background region and Profile $>0.55$ constituted the signal region. The background in the $\tau$ signal region was estimated using a QCD sample. Table 2 details the event number breakdown. The cross section is $\sigma \cdot B\left(W \rightarrow \tau \nu_{\tau}\right)=2.38 \pm 0.09 \pm 0.10 \mathrm{nb}$, where the luminosity uncertainties have not been included. This gives a coupling relative to that for the electron of $g_{\tau}^{W} / g_{e}^{W}=1.00 \pm 0.02 \pm 0.03$; consistent with lepton universality.

\section{DRELL-YAN COMPOSITENESS LIMITS}

CDF has searched in Drell-Yan production at the Tevatron for evidence of quark or lepton compositeness. Substructure would add a contact term to the dilepton production amplitude and manifest itself mainly in the interference with the Standard Model amplitude. Interaction strengths for the contact term are assumed to be of the form, $\xi_{i j}= \pm g_{0}^{2} / \Lambda_{i j}^{2}$, where $i, j$ are the helicities. The compositeness scale, $\Lambda_{i j}$, is defined assuming $g_{\circ}^{2} / 4 \pi=1$. The 
TABLE 2. D $\emptyset \rightarrow \tau \nu_{\tau}$ Results

\begin{tabular}{c|c}
\hline Event Source & Number of Events \\
\hline Final Data Sample & 1202 \\
QCD Background & $106 \pm 7 \pm 5$ \\
Noise Events & $81 \pm 14$ \\
$Z \rightarrow \tau^{+} \tau^{-}$ & $32 \pm 5$ \\
$W \rightarrow e \nu_{e}$ & $3 \pm 1$ \\
\hline
\end{tabular}

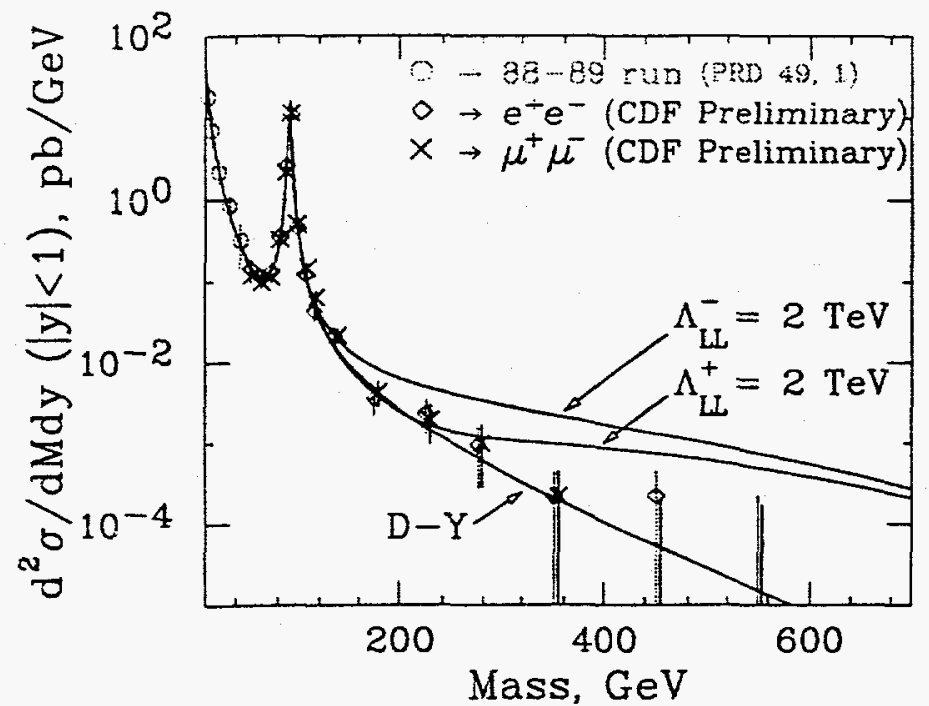

FIGURE 8. The differential mass cross section for a tightly selected isolated lepton plus a second lepton with looser selection criteria. QCD background was estimated from same sign lepton pairs while $W W, \tau \tau, c \bar{c}$, etc. backgrounds were estimated from $e \mu$ pairs.

dilepton differential mass spectrum for rapidity less than 1 is plotted and normalized to the region around $M_{Z}$, specifically $50<M\left(\ell^{+} \ell^{-}\right)<150 \mathrm{GeV} / \mathrm{c}^{2}$. A binned likelihood is calculated for the region $M\left(\ell^{+} \ell^{-}\right)>150 \mathrm{GeV} / \mathrm{c}^{2}$ and $95 \%$ confidence level limits are determined for a contact term for a variety of helicity assumptions. Figure 8 shows the mass spectrum along with expectations for a totally left-handed contact term at a compositeness scale of $2 \mathrm{TeV}$. Assuming quark and lepton universality in compositeness, limits on $\Lambda^{+}$(positive sign of coupling) range from $>3.0-5.0 \mathrm{TeV}$, while for $\Lambda^{-}$the range is $>3.7-6.3 \mathrm{TeV}$ for a compositeness scale.

\section{SUMMARY}

The Tevatron run $1 \mathrm{~b}$ integrated luminosity increase of 5-6 over run 1a halved the uncertainty of $M_{W}$. D $\varnothing$ has significantly tightened limits on anomalous 
gauge boson couplings; especially from analysis of the $Z \rightarrow \nu \bar{\nu}$ decay channel. Run $1 \mathrm{~b}$ has also witnessed the first observation of $W W, W Z$, and $Z Z$ production. Pseudorapidity region coverage for the $W$ charge asymmetry has doubled to $|\eta| \sim 2.2$ providing improved constraints on parton distribution functions. $D \emptyset$ has demonstrated lepton universality through the measurement of $\sigma \cdot B\left(W \rightarrow \tau \nu_{\tau}\right)$. Analysis of Drell-Yan production has allowed CDF to push the compositeness scale limits to greater than 3-6 $\mathrm{TeV}$ depending on the helicity and sign of the coupling assumed.

In run 2, $D \varnothing$ and $C D F$ will continue precision electroweak sector measurements. The $W$ mass uncertainty should be reduced to near $\pm 40 \mathrm{MeV} / \mathrm{c}^{2}$. Limits on anomalous gauge boson self-couplings will continue to improve and observation of the radiation amplitude zero in $W \gamma$ production [11] will be addressed.

I thank the organizers of the Beyond the Standard Model V conference for a well run, informative, and enjoyable conference in a beautiful location. I am indebted to the members of the $D \emptyset$ and CDF electroweak physics groups for help in obtaining the latest results and plots, and for comments on the manuscript.

\section{REFERENCES}

1. The most current electroweak results are available on the public World Wide Web page each experiment maintains:

CDF - www-cdf.fnal.gov/physics/ewk/ewk.html $D \emptyset$ - www-d0.fnal.gov/public/wz/ewk_public.html

2. Alitti, J. et al. , Phys. Lett. B 241, 150 (1990).

3. Abe, F. et al., Nucl. Instrum. Methods A 271, 387 (1988); Abe, F. et al. Phys. Rev. D 50, 2966 (1994); Amidei, D. et al. Nucl. Instrum. Methods A 350, 73 (1994).

4. Abachi, S. et a!. , Nucl. Instrum. Methods $A$ 338, 185 (1994).

5. Particle Data Group: Barnett, R. M. et al. , Phys. Rev. D 54 Part I, 530 (1996).

6. Arnold, P. B. and R. P. Kauffman, Nucl. Phys. B 349, 381 (1991).

7. Ladinsky, G. A. and C.-P. Yuan, Phys. Rev. D 50, 4239 (1994).

8. Hagiwara, K. et al., Nucl. Phys. B 282, 253 (1987).

9. Baur, U. and E. L. Berger, Phys. Rev. D 47, 4889 (1993).

10. Abe, F. et al., Phys. Rev. Lett. 68, 3398 (1992).

11. Brown, R. W., K. Kowalski, and S. J. Brodsky, Phys. Rev. D 28, 624 (1983). 